\title{
Experiencia en cirugía de catarata asistida por láser de femtosegundo en la Fundación Hospital Nuestra Señora de la Luz
}

\author{
Experience in cataract surgery assisted by femtosecond laser at Fundación Hospital \\ Nuestra Señora de la Luz
}

\author{
Sandra N. Chimalpopoca-Bautista*, Cyntia Solís-Hernández y Claudia Palacio-Pastrana \\ Departamento de Segmento Anterior, Fundación Hospital Nuestra Señora de la Luz, Ciudad de México, México
}

\begin{abstract}
Resumen
Objetivo: Describir las complicaciones transoperatorias de las cirugías de catarata asistidas por láser de femtosegundo. Método: Estudio observacional, descriptivo, retrospectivo y transversal de 420 expedientes de cirugías realizadas con la plataforma LenSx de abril de 2015 a agosto de 2017. La revisión de expedientes se realizó a través del sistema electrónico interno accediendo a las notas preoperatoria y posoperatoria. La información se recopiló en una base de datos Excel. Se realizó el análisis de variables por medio de estadística descriptiva con medidas de tendencia central. Resultados: Cumplieron criterios de inclusión 86 expedientes. La edad promedio de los pacientes fue de 63 años. Se presentó una frecuencia general de complicaciones del $27.9 \%$ (24 cirugías). Las complicaciones que ocurrieron con mayor frecuencia fueron herida corneal incompleta o impermeable (37.5\%), capsulorrexis incompleta (25\%) y rotura de cápsula posterior (16.7\%). Conclusiones: La tecnología de láser de femtosegundo incorporada en la cirugía de catarata puede considerarse como exitosa al tener una baja frecuencia general de complicaciones.
\end{abstract}

Palabras Clave: Cirugía de catarata. Láser de femtosegundo. Complicaciones.

\begin{abstract}
Objective: To describe the transoperative results of cataract surgeries assisted by femtosecond laser. Method: Observational, descriptive, retrospective and cross-sectional study of 420 surgical records made with the LenSx platform from April 2015 to August 2017. The review of records was made through the internal electronic system accessing the preoperative and postoperative note. The information was collected through a database in Excel. The analysis of variables was performed by means of descriptive statistics with measures of central tendency. Results: 86 files met inclusion criteria. The average age was 63 years. There was a general frequency of complications of $27.9 \%$ (24 surgeries). The most frequent complications were incomplete or impermeable corneal wound (37.5\%), incomplete capsulorhexis (25\%) and posterior capsule rupture (16.7\%). Conclusions: The femtosecond laser technology incorporated in cataract surgery can be considered successful because of a low overall frequency of complications.
\end{abstract}

Key Words: Cataract surgery. Femtosecond laser. Complications.

\footnotetext{
Correspondencia:

*Sandra N. Chimalpopoca-Bautista

Ezequiel Montes, 135

Col. Tabacalera, Del. Cuauhtémoc

C.P. 06030, Ciudad de México, México

Fecha de recepción: 09-06-2019

Cir Cir. 2020;88(3):321-324

E-mail: sandran.chb@gmail.com

Fecha de aceptación: 22-01-2020

DOI: 10.24875/CIRU.20001359

Contents available at PubMed

www.cirugiaycirujanos.com

0009-7411/@ 2020 Academia Mexicana de Cirugía. Publicado por Permanyer. Este es un artículo open access bajo la licencia CC BY-NC-ND (http://creativecommons.org/licenses/by-nc-nd/4.0/).
} 


\section{Introducción}

En el mundo existen aproximadamente 285 millones de personas con discapacidad visual, de las cuales 39 millones son ciegas y 246 millones presentan baja visión, siendo las cataratas la principal causa de ceguera en los países de ingresos medios y bajos. Se estima que las cataratas no operadas representan el $33 \%$ de la discapacidad visual mundial ${ }^{1}$.

La técnica de facoemulsificación convencional para la extracción de catarata ha sido desplazada por la utilización de láser de alta frecuencia asociado a tecnología de imagen ocular, que provee una precisión quirúrgica que difícilmente podría obtenerse con la técnica manual².

La Food and Drug Administration aprobó inicialmente el uso del sistema de láser de femtosegundo para incisiones corneales y capsulorrexis anterior, y en abril de 2010 se aprobó su uso para fragmentación de catarata ${ }^{2}$.

El láser de femtosegundo funciona en una longitud de onda en el infrarrojo cercano $(1030 \mathrm{~nm})$. El mecanismo de acción básico radica en el hecho de que un haz de energía altamente enfocado puede elevar la energía de la materia a un nivel donde se crea el plasma. Este plasma da electrones libres y moléculas ionizadas se expande rápidamente, creando burbujas de cavitación. La fuerza de creación de burbujas de cavitación separa el tejido. Este proceso de convertir la energía del láser en energía mecánica se conoce como fotodisrupción. La fotodisrupción intraocular está guiada por sistemas de imagen que utilizan la tomografía de coherencia óptica (OCT) en tiempo real, que permite definir con precisión las estructuras del segmento anterior hasta la cápsula posterior, por lo que también puede usarse para apuntar a la cámara anterior a varias profundidades sin riesgo de daño colateral. El procedimiento quirúrgico con esta tecnología comienza con el ajuste de un anillo de succión en el ojo que a su vez está unido al sistema óptico del láser. En este momento, la OCT captura el segmento anterior en una sola imagen, proyectada en la pantalla con capacidad para la manipulación tridimensional usando el software del dispositivo. Las incisiones se ubican con respecto a su ancho, profundidad, perfil y longitud. Con estos datos determinados por el software se inicia el procedimiento presionando el pedal. La intervención es visible en tiempo real mientras se realiza. Con esta tecnología es posible realizar incisiones corneales, capsulotomía anterior y fragmentación del núcleo ${ }^{3,4}$.
Con el láser de femtosegundo se consiguen incisiones corneales en túnel y con la longitud requerida, pudiendo elegir la forma cuadrada, que se ha demostrado que es más resistente a la deformación y a la fuga 5 .

Durante la capsulotomía, el láser de femtosegundo nos permite obtener trazos regulares, centrados en la posición deseada con un borde definido y rectilíneo, lo que aumenta su resistencia y la seguridad al realizar la fragmentación del núcleo y la inserción de la lente intraocular ${ }^{6}$. Para la fragmentación, el láser de femtosegundo realiza en el cristalino el patrón de corte que más interese según el tipo de catarata de que se trate, modificando los parámetros de localización, forma, número y distancia a la cápsula posterior y anterior, consiguiendo así una fragmentación previa del núcleo totalmente personalizada para cada paciente y un tiempo más corto de aplicación de ultrasonidos, que podría conducir a una menor inflamación intraocular posquirúrgica y a menos pérdida de células endoteliales 7,8 .

En México, el Hospital Central Militar reportó su experiencia a lo largo de 2 años estableciendo diferencias en la técnica estándar del femtofaco, como son los límites para la fractura del cristalino, el corte epitelial por arriba del plano corneal para lograr mayor porcentaje de apertura de incisión principal y la modificación en la secuencia de fractura e hidrodisección para evitar un bloqueo capsular, considerando estas variables como fundamentales para optimizar los resultados de la técnica y acortar la curva de aprendizaje que esta puede representar ${ }^{9}$.

La integración del láser de femtosegundo a la cirugía de catarata es una técnica que continúa en investigación clínica respecto a su eficacia, seguridad, ventajas y limitaciones. El presente estudio tiene como objetivo describir los resultados de la técnica quirúrgica de cirugía de catarata asistida por femtosegundo en la Fundación Hospital Nuestra Señora de la Luz, de Ciudad de México.

\section{Método}

Se realizó un estudio observacional, descriptivo, retrospectivo y transversal. Se incluyeron expedientes de pacientes sometidos a facoemulsificación asistida por láser de femtosegundo sin opacidades corneales, con hendiduras palpebrales y queratometrías que permitieran un acoplamiento adecuado. La nota posoperatoria debía documentar claramente el uso de láser de femtosegundo, así como hallazgos y complicaciones; de 
lo contrario, los expedientes eran excluidos. Así mismo, fueron excluidos los pacientes con cataratas totales hidratadas. Se revisaron 420 expedientes de casos de cirugía de catarata asistida con láser de femtosegundo realizadas en el Departamento de Segmento Anterior de la Fundación Hospital Nuestra Señora de la luz, IAP con el LenSx de la casa comercial Alcon, entre abril de 2014 y agosto de 2017.

Las variables analizadas durante el estudio fueron la permeabilidad de la herida, los desgarros de la cápsula anterior, la capsulorrexis incompleta, la fractura del núcleo incompleta, la rotura de cápsula posterior, la diálisis zonular y la miosis posfemto.

La revisión se llevó a cabo a través del sistema de expediente clínico electrónico con el que cuenta nuestro centro hospitalario, accediendo a las notas preoperatoria y posoperatoria. Se recopiló la información en una base de datos Excel y se analizó cada variable por medio de estadística descriptiva con medidas de tendencia central.

\section{Resultados}

De los 420 expedientes revisados, 86 cumplieron criterios de inclusión. Se realizaron 29 cirugías en 2015, 29 en 2016 y 28 hasta abril de 2017. La edad promedio de los pacientes fue de 63 años. Hubo $53(61.63 \%)$ mujeres y $33(38.37 \%)$ hombres. De los pacientes incluidos, 47 (54.65\%) presentaban comorbilidad (18 hipertensión, 15 diabetes y 14 ambas). La frecuencia general de complicaciones se muestra en la figura 1: el $72.1 \%$ de las cirugías no reportaron ninguna complicación, mientras que el $27.9 \%$ tuvieron alguna. La complicación más frecuente fue la herida corneal incompleta, con nueve casos, seguida de la capsulorrexis incompleta, con seis casos. El total de las complicaciones muestra en la figura 2.

\section{Discusión}

El láser de femtosegundo incorporado a la cirugía de catarata ha sido utilizado en diversos centros hospitalarios. Como se muestra en la tabla 1, la frecuencia de complicaciones que obtuvimos difiere de lo reportado en la literatura. Roberts, et al..$^{10} \mathrm{y}$ Chang, et al. ${ }^{11}$ hallaron una mayor frecuencia de desgarro de cápsula anterior y de miosis posfemto, contrario a lo reportado por Abell, et al. ${ }^{7,8}$, quienes no presentaron ningún caso de desgarro de cápsula a anterior y solo un $3 \%$ de miosis. En nuestro caso los desgarros fueron ecuatoriales, por lo que se logró completar la

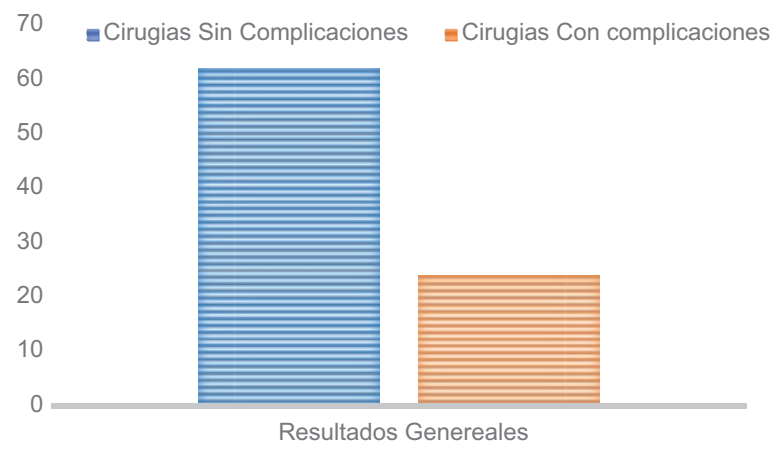

Figura 1. Frecuencia general de complicaciones: 62 cirugías sin complicaciones y 24 cirugías con complicaciones.

Tabla 1. Complicaciones reportadas en comparación con otras series

\begin{tabular}{lcccc}
\hline & FHNSL & $\begin{array}{c}\text { Roberts, } \\
\text { et al. }\end{array}$ & $\begin{array}{c}\text { Chang, } \\
\text { et al. }{ }^{11}\end{array}$ & $\begin{array}{c}\text { Abell, } \\
\text { et al. }{ }^{7.8}\end{array}$ \\
\hline $\begin{array}{l}\text { Herida corneal } \\
\text { incompleta }\end{array}$ & $9(37.5 \%)$ & & & \\
$\begin{array}{l}\text { Desgarro de } \\
\text { cápsula anterior }\end{array}$ & $2(8.3 \%)$ & $10.5 \%$ & $5.3 \%$ & $0 \%$ \\
$\begin{array}{l}\text { Capsulorrexis } \\
\text { incompleta }\end{array}$ & $6(25 \%)$ & & & \\
$\begin{array}{l}\text { Miosis } \\
\begin{array}{l}\text { Fractura del núcleo } \\
\text { incompleta }\end{array}\end{array}$ & $2(8.3 \%)$ & $9.5 \%$ & $10 \%$ & $3 \%$ \\
$\begin{array}{l}\text { Rotura de cápsula } \\
\text { posterior }\end{array}$ & $4(16.7 \%)$ & $3.5 \%$ & & $0.5 \%$ \\
\begin{tabular}{l} 
Diálisis zonular \\
\hline
\end{tabular} & $1(4.2 \%)$ & & & \\
\hline
\end{tabular}

FHNSL: Fundación Hospital Nuestra Señora de la Luz.

capsulotomía manualmente. La complicación que se presentó con mayor frecuencia fue la herida corneal incompleta, la cual se completó manualmente con cuchilletes permitiendo la realización de los siguientes pasos de la facoemulsificación sin inconvenientes. La complicación intraoperatoria más seria fue la rotura de cápsula posterior, que tuvo una proporción mayor en nuestro centro hospitalario, como se muestra en la tabla 1, requiriendo una vitrectomía anterior y en todos los casos se colocó un lente de tres piezas en el sulcus. Las complicaciones reportadas son en su mayoría susceptibles de ser resueltas con técnicas manuales sin deterioro en el pronóstico visual del paciente, por lo que es necesario que el cirujano que utilice la tecnología de láser de femtosegundo domine la técnica manual. Ya que la técnica es dependiente del operador, es recomendable que se calibren los aparatos y se ajusten los parámetros 


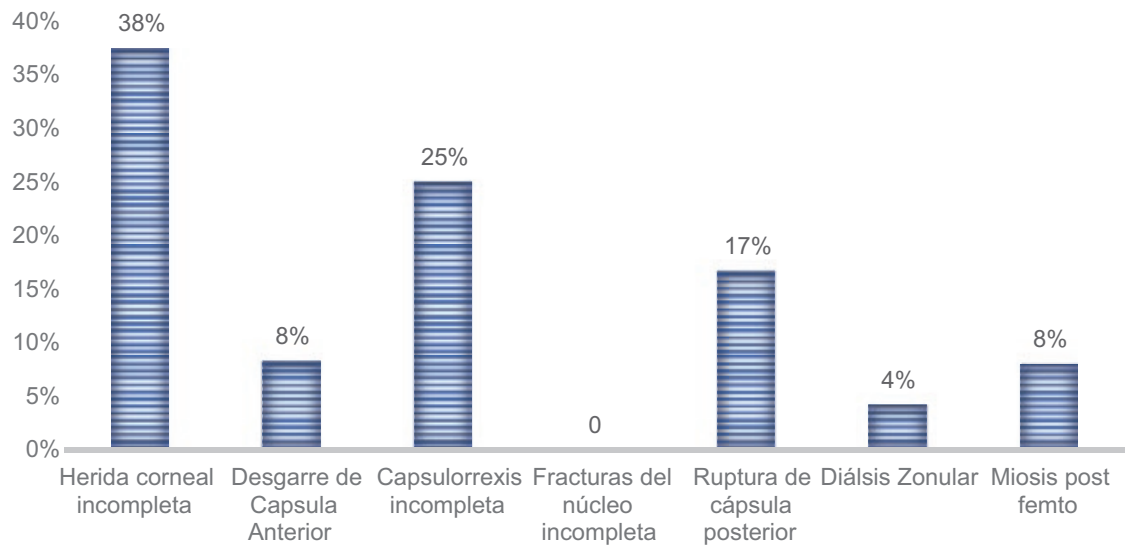

Figura 2. Distribución de las complicaciones.

en cuanto a entrega de energía, dimensión y profundidad de las heridas de acuerdo con la experiencia en los casos iniciales del cirujano. En la comparación de resultados es necesario tomar en cuenta que la tasa de complicaciones reportada depende de la experiencia del cirujano y de si la serie fue de un cirujano único o de varios, o si es un estudio multicéntrico. En nuestro estudio, las cirugías fueron realizadas tanto por cirujanos experimentados como por cirujanos en formación que cumplían su curva de aprendizaje.

El láser de femtosegundo permanece en la práctica como una técnica vanguardista que continúa implementando modificaciones al sistema, como interfaces para curvaturas corneales que salen del rango de normalidad, permitiendo aumentar el número de pacientes tratados y disminuir la tasa de complicaciones.

\section{Conclusión}

La cirugía de catarata asistida por láser de femtosegundo es un procedimiento exitoso con una baja frecuencia de complicaciones que requiere una curva de aprendizaje. Esperamos que los resultados del presente estudio sirvan para evaluar el impacto que tiene el láser de femtosegundo en la cirugía de catarata.

\section{Agradecimientos}

Al Dr. Jaime Lozano Alcázar y la Dra. Adriana Saucedo Castillo.

\section{Conflicto de intereses}

Los autores declaran no tener conflicto de intereses.

\section{Responsabilidades éticas}

Protección de personas y animales. Los autores declaran que para esta investigación no se han realizado experimentos en seres humanos ni en animales.

Confidencialidad de los datos. Los autores declaran que han seguido los protocolos de su centro de trabajo sobre la publicación de datos de pacientes.

Derecho a la privacidad y consentimiento informado. Los autores han obtenido el consentimiento informado de los pacientes y/o sujetos referidos en el artículo. Este documento obra en poder del autor de correspondencia.

\section{Bibliografía}

1. Asamblea Mundial de la Salud, 66. Proyecto de plan de acción para la prevención de la ceguera y la discapacidad visual evitables 2014-2019. Salud ocular universal: un plan de acción mundial para 2014-2019. Informe de la Secretaría; 2013.

2. Potvin, R, Makari, S. Cataract surgery and the LenSx® Femtosecond Laser System. US Ophthalmic Review. 2013;6:89-93.

3. Alió JL. Cataract surgery with femtosecond lasers. Saudi J Ophthalmol. 2011;25:219-23.

4. Ali MH, Javaid M, Jamal S, Butt NH. Femtosecond laser assisted cataract surgery, beginning of a new era in cataract surgery. Oman J Ophthalmol. 2015;8:141-6.

5. Masket S, Sarayba M, Ignacio T, Fram N. Femtosecond laser-assisted cataract incisions: architectural stability and reproducibility. J Cataract Refract Surg. 2010;36:1048-9.

6. Friedman NJ, Palanker DV, Schuele G, Andersen D, Marcellino G, Seibel BS, et al. Femtosecond laser capsulotomy. J Cataract Refract Surg. 2011;37:1189-98.

7. Abell RG, Allen PL, Vote BJ. Anterior chamber flare after femtosecond laser-assisted cataract surgery. J Cataract Refract Surg. 2013;39:1321-6.

8. Abell RG, Kerr NM, Vote BJ. Femtosecond laser-assisted cataract surgery compared with conventional cataract surgery. Clin Exp Ophthalmol. 2013:41:455-62

9. Moya Romero JO, Cantero Vergara MA, Gómez Cortés CA. Cirugía de catarata asistida con láser de femtosegundo. Técnica Hospital Central Militar México. Rev Mex Oftalmol. 2016;90:49-56.

10. Roberts TV, Lawless M, Bali SJ, Hodge C, Sutton G. Surgical outcomes and safety of femtosecond laser cataract surgery: a prospective study of 1500 consecutive cases. Ophthalmology. 2013;120:227-33.

11. Chang JS, Chen IN, Chan WM, Ng JC, Chan VK, Law AK. Initial evaluation of a femtosecond laser system in cataract surgery. J Cataract Refract Surg. 2014;40:29-36. 